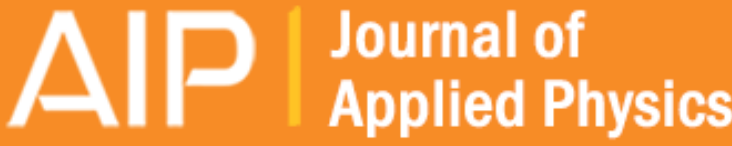

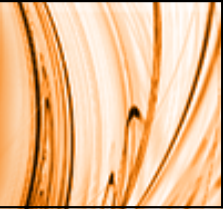

\section{lonic polymer cluster energetics: Computational analysis of pendant chain stiffness}

and charge imbalance

Lisa Mauck Weiland and Donald J. Leo

Citation: Journal of Applied Physics 97, 123530 (2005); doi: 10.1063/1.1937475

View online: http://dx.doi.org/10.1063/1.1937475

View Table of Contents: http://scitation.aip.org/content/aip/journal/jap/97/12?ver=pdfcov

Published by the AIP Publishing

\section{Articles you may be interested in}

Microwave and mechanical properties of quartz/graphene-based polymer nanocomposites

Appl. Phys. Lett. 102, 072903 (2013); 10.1063/1.4793411

Monitoring progressive damage in polymer-based composite using nonlinear dynamics and acoustic emission J. Acoust. Soc. Am. 125, EL39 (2009); 10.1121/1.2993755

Modeling of electrochemomechanical response of ionic polymer-metal composites with various solvents J. Appl. Phys. 100, 064310 (2006); 10.1063/1.2221505

Computational analysis of ionic polymer cluster energetics

J. Appl. Phys. 97, 013541 (2005); 10.1063/1.1819974

Micromechanics of actuation of ionic polymer-metal composites

J. Appl. Phys. 92, 2899 (2002); 10.1063/1.1495888

\section{MIT LINCOLN} LABORATORY CAREERS

Discover the satisfaction of innovation and service to the nation
- Space Control

- Air \& Missile Defense

- Communications Systems \& Cyber Security

- Intelligence, Surveillance and

Reconnaissance Systems
- Advanced

Electronics

- Tactical Systems

- Homeland

Protection

- Air Traffic Control






\title{
Ionic polymer cluster energetics: Computational analysis of pendant chain stiffness and charge imbalance
}

\author{
Lisa Mauck Weiland ${ }^{\text {a) }}$ and Donald J. Leo \\ Center for Intelligent Material Systems and Structures, Mechanical Engineering Department, Virginia Tech, \\ Blacksburg, Virginia 24061-0261
}

(Received 22 July 2004; accepted 25 April 2005; published online 23 June 2005)

\begin{abstract}
In recent years there has been considerable study of the potential mechanisms underlying the electromechanical response of ionic-polymer-metal composites. The most recent models have been based on the response of the ion-containing clusters that are formed when the material is synthesized. Most of these efforts have employed assumptions of uniform ion distribution within spherical cluster shapes. This work investigates the impact of dispensing with these assumptions in order to better understand the parameters that impact cluster shape, size, and ion transport potential. A computational micromechanics model applying Monte Carlo methodology is employed to predict the equilibrium state of a single cluster of a solvated ionomeric polymer. For a constant solvated state, the model tracks the position of individual ions within a given cluster in response to ion-ion interaction, mechanical stiffness of the pendant chain, cluster surface energy, and external electric-field loading. Results suggest that cluster surface effects play a significant role in the equilibrium cluster state, including ion distribution; pendant chain stiffness also plays a role in ion distribution but to a lesser extent. Moreover, ion pairing is rarely complete even in cation-rich clusters; this in turn supports the supposition of the formation of anode and cathode boundary layers. (c) 2005 American Institute of Physics. [DOI: 10.1063/1.1937475]
\end{abstract}

\section{INTRODUCTION}

It has been over a decade since the transduction properties of ionic-polymer-metal composites (IPMCs) were observed. ${ }^{1-5}$ In that time there has been considerable conjecture over the potential applications of these soft transducers ${ }^{2,3,6-8}$ and considerable fruitful investigation towards these ends. ${ }^{9-11}$ However, many aspects of their electromechanical behavior still are not understood, impeding full exploitation of these devices. For instance, because these devices (for most polymer and associated counterion forms) display a fast response followed by a slow "relaxation" response when fully solvated, it is clear that at least two different electromechanical response mechanisms are stimulated by the application of an electric field. The nature of these mechanisms is still under investigation.

Most models of the transduction behavior rely on the cluster morphology of the ionic polymer layer as proposed by Hsu and Gierke, ${ }^{12}$ where the material has been solvated with water. In brief, the backbone of the ionic-polymer chain is hydrophobic while the side chains terminate in hydrophilic ionic groups. Hsu and Gierke propose that these hydrophilic ionic side groups and the water that has been taken up by the material cluster together. The model further suggests an idealized structuring whereby the clusters are of essentially constant radius, uniformly distributed throughout the material, and interconnected by channels. Subsequently, many have sought to quantify the cluster radius and spacing as functions of polymer equivalent weight, hydration level, counterion,

\footnotetext{
$\overline{\left.{ }^{a}\right)}$ Electronic mail: 1weiland@engr.pitt.edu
}

etc. See, for instance, the modeling works of Datye and co-workers $^{13,14}$ and the experimental work of Lehmani et al. ${ }^{15}$

Early transduction models proposed an entirely hydraulic description of the electromechanical response. In this approach counterions and water migrate through the thickness of the material in response to electric field; this in turn results in differential swelling of the clusters and the observed bending response. Because these models are consistent with a substantial proportion of the experimentally observed response, considerable strides have been made in the effort to develop them. ${ }^{16}$ However, it has since been noted that these models are in conflict with certain aspects of recently obtained experimental observations. ${ }^{8}$ A subsequent hybrid model proposed by $\mathrm{Li}$ and Nemat-Nasser ${ }^{17}$ and NematNasser and $\mathrm{Li}^{18}$ addresses the above-noted multimechanisms by considering the interplay of multiple contributions to the overall energy state including elastic, electrostatic, and osmotic effects. The first of these efforts ${ }^{17}$ focuses primarily on the clustering, in which they conclude the clusters within the ionomer studied will be oblate in the presence of an applied electric field, and spherical in its absence. In each of the efforts ${ }^{17,18}$ they also assume uniform and paired distribution of the ionic side groups over the surface of each spherical cluster, while in the later effort, ${ }^{18}$ the formation of anode and cathode boundary layers during IPMC actuation is hypothesized.

Based in part on the aforementioned studies by NematNasser and $\mathrm{Li}$, as well as the results of earlier works by the current authors, ${ }^{19,20}$ this effort seeks to address the impact of pendant chain stiffness and charge imbalance as they may relate to the formation of electrode boundary layers. In the 
first of these works, ${ }^{19}$ results suggest that the pendant chain stiffness, cluster surface boundary, and locally increased electric field may play significant roles in boundary layer response. In the subsequent, more detailed study ${ }^{20}$ a Monte Carlo style computational approach is developed and applied to the identification of cluster equilibrium states in order to assess the assumption of uniform charge distribution, perfectly spherical cluster shape, and the impact of varying cluster surface tension. One significant result of that work is the prediction of incomplete pairing; this prediction not only suggests that the clusters within an ionomer are predisposed to ion transport, but also suggests that application of the assumption of uniform and paired ion distribution will lead to an underprediction of IPMC actuation response. A second significant result of that work is the significant role that the cluster surface plays in the predicted equilibrium state.

In the present work a Monte Carlo style computational approach is applied to the study of cluster equilibrium states to assess the impact of varying pendant chain stiffness and charge imbalance; introduction of an energy barrier to ion motion is also considered. The intent is to facilitate the application of larger length scale models, such as those noted above, through identification of sources of error and their impact, as well as facilitate ionomer fabrication and experimental studies aimed at optimizing the transduction response. In this effort no assumption of uniform ion distribution is required, and subsequent response may lead to nonspherical cluster shapes. Ion movement occurs in response to ion-ion interactions, elastic constraint of the pendant chains, cluster surface energy, and externally applied electric field.

The paper is organized as follows. Section II discusses the theoretical basis and construction of the model. Section III provides model predictions for a range of parametric studies. Section IV presents discussion of the predictions. Section $\mathrm{V}$ concludes the work.

\section{MODELING APPROACH}

This work applies the modeling approach developed and validated by Weiland and Leo. ${ }^{20}$ This section provides a brief review of the approach. The approach is focused on understanding the interactions within the clusters that are formed in ionomer materials. These clusters consist of an aggregation of charged side groups, counterions, and solvent, and it is commonly believed that a network of clusters is connected through "channels" that facilitate the transport of the counterion through the polymer. It is this ion conduction property that gives rise to electromechanical transduction.

Our goal is to understand the interactions of ions within a cluster as a function of relevant polymer properties as illustrated in Fig. 1. The cluster model consists of a specified number of negatively charged ionic groups ("anions"), and positively charged counterions ("cations"). We assume that the cluster is solvated with a liquid and that this liquid creates a surface tension at the boundaries of the cluster which will restrict the motion of cations and anions. Further, we assume that this cluster surface, or hydrophobic-hydrophilic boundary, is defined by the location of the anions.



FIG. 1. Identification of the region of study (a single cluster) as it relates to an IPMC.

This development is based on static energy states and assumes a reversible process, where losses due to solvent viscosity and pendant chain reconfiguration are fundamentally dynamic processes. This model concerns itself with identifying the low-energy state of a cluster for a specified distribution of ions, and thus no transport of ions outside the cluster is considered. Transport is considered indirectly through identification of the low-energy state with variations in charge imbalance; the impact of osmotic-pressure variations due to water transport is not considered so that electrostatic contribution to transduction response may be considered in isolation. The introduction of cluster charge imbalance necessarily results in a local, nonzero charge density. In this work the charge density is calculated over the spherical volume defined by the ion with the largest radial position.

We hypothesize the existence of cations located outside the anion boundary shell, which are termed "outer cations" in this work. Further, we define ion pairing as the close proximity of a cation and an anion. Unlike some previous models which have assumed that ion pairing is complete, the present model allows the existence of "unpaired ions," i.e., those anions that are not in close proximity to a cation.

\section{A. Theory}

The model is constructed such that the ions are allowed to move independently in a manner governed by the summation of electrostatic, elastic, and cluster surface forces, where this summation dictates the proposed stepping direction. A 
step in the same direction and of maximum magnitude $0.002 \mathrm{~nm}$ is tested. ${ }^{20}$ If the step leads to ion overlap, the step size is reduced or rejected depending on the proximity of the conflicting ion. The step is then tested to confirm that it leads to a lower-energy state via the summation of corresponding energy terms; if it fails this test the step is rejected. In both summations, it is assumed that no direct coupling exists among the critical terms. The system is considered converged only when all ion steps are rejected. These summations are given by

$$
\begin{aligned}
& \mathbf{F}_{\text {tot }}=\mathbf{F}_{\text {ext }}+\mathbf{F}_{\text {ion-ion }}+\mathbf{F}_{\mathrm{pc}}+\mathbf{F}_{s}, \\
& U_{\text {tot }}=U_{\text {ext }}+U_{\text {ion-ion }}+U_{\mathrm{pc}}+U_{s},
\end{aligned}
$$

where the subscript "ext" refers to response driven by externally applied electric field, "ion-ion" refers to electrostatic ion-ion interactions, "pc" refers to pendant chain response to motion of the attached anion, and $s$ refers to the interaction of ions with the cluster surface. In the application of Eqs. (1) and (2), each ion is considered individually. Convergence (equilibrium) of the system is reached when all ion steps are rejected as inadmissible, thus the total system energy has been minimized for the given step size.

The force exerted on any given ion due to the total external electric field is found by multiplying the charge of that ion, $c$, by the externally applied field.

$$
\mathbf{F}_{\mathrm{ext}}=c \mathbf{E}_{\mathrm{ext}} \text {. }
$$

The corresponding change in the potential energy associated with ion movement due to uniform external fields is given by

$$
U_{\mathrm{ext}}=-\mathbf{F}_{\mathrm{ext}} \cdot \mathbf{p},
$$

where $\mathbf{p}$ is the ion position in three-dimensional space.

Similarly, the force exerted on any ion due to every other ion may be expressed as

$$
\mathbf{F}_{\text {ion-ion }}=c \mathbf{E}_{\text {ion-ion }} \text {. }
$$

$\mathbf{E}_{\text {ion-ion }}$ is given by

$$
\begin{aligned}
\mathbf{E}_{\text {ion-ion }} & =\sum_{j=1, j \neq i}^{\left(n_{\text {an }}+n_{\text {cat }}\right)} E_{i-j} \\
& =\sum_{j=1, j \neq i}^{\left(n_{\text {an }}+n_{\text {cat }}\right)} \frac{9 \times 10^{9} c_{j}}{\varepsilon \cdot r_{i-j}^{3}}\left(1-\left(\frac{r_{o}}{r_{i-j}}\right)^{10}\right) \cdot \mathbf{r}_{i-j},
\end{aligned}
$$

where $\mathbf{E}_{i-j}$ is the electric field acting on ion $i$ due to ion $j, \varepsilon$ is the net effective dielectric constant, $c_{j}$ is the charge of the $j$ th ion, $r_{o}$ is the equilibrium separation between atoms, and $\mathbf{r}_{i-j}$

$$
\mathbf{r}_{i-j}=\mathbf{p}^{i}-\mathbf{p}^{j},
$$

is the vector distance separating ion $i$ from ion $j$, while $r_{i-j}$ is the magnitude of $\mathbf{r}_{i-j}$.

The first parenthetical term of Eq. (6) is consistent with classic electrostatic theory. ${ }^{21}$ The second parenthetical term derives from the nature of an ionic bond where Pauli repulsion prevents overlapping ion clouds and the exponential value is found experimentally to vary between 3 and 12..$^{20,22,23}$ By applying this expression, cation-anion pairs necessarily respond in a manner consistent with dipole response without having to explicitly account for the pairing.

The corresponding potential for ion-ion interaction is given by

$$
\begin{aligned}
U_{\text {ion-ion }} & =\sum_{j=1, j \neq i}^{\left(n_{\text {an }}+n_{\text {cat }}\right)} U_{i-j} \\
& =\sum_{j=1, j \neq i}^{\left(n_{\text {an }}+n_{\text {cat }}\right)} \frac{9 \times 10^{9} c_{j} c_{i}}{\varepsilon}\left(\frac{1}{r_{i-j}}-\frac{1}{11 r_{i-j}} \cdot\left(\frac{r_{o}}{r_{i-j}}\right)^{10}\right) .
\end{aligned}
$$

The resistance/assistance to anion motion due to the covalent bond to the pendant chain is approximated by a linear reversible spring, such that

$$
\mathbf{F}_{\mathrm{pc}}=k_{\mathrm{pc}} \mathbf{x}=-k_{\mathrm{pc}}\left(\mathbf{p}-\mathbf{p}_{o}\right)
$$

where $\mathbf{p}_{o}$ is the initialized anion position and $k_{\mathrm{pc}}$ is estimated from the models of Datye and co-workers. ${ }^{13,14}$ For the case of constant $k_{\mathrm{pc}}$, where it is understood that a constant pendant chain stiffness represents a simplifying assumption, the change in energy associated with anion displacement from position $\mathbf{p}_{o}$ to position $\mathbf{p}$ is given by

$$
U_{\mathrm{pc}}=\frac{1}{2} k_{\mathrm{pc}}\left(\mathbf{p}-\mathbf{p}_{o}\right) \cdot\left(\mathbf{p}-\mathbf{p}_{o}\right)
$$

The surface energy of the cluster is the mechanism which separates the hydrophobic and hydrophilic regions in ionic polymer morphology. In the absence of all external stimuli (gravity, ion motion, etc.) the cluster shape minimizing cluster surface energy is spherical. However, because the cluster surface acts as the hydrophobic/hydrophilic barrier, ion-surface interaction can distort this shape away from this spherical, low-energy state. The expression for the force imposed on an ion due to cluster surface distortion is given by

$$
\mathbf{F}_{s}=-2 \gamma \pi\left[\|\mathbf{p}\|-r_{\mathrm{cl}}\right] \cdot \frac{\mathbf{p}}{\|\mathbf{p}\|},
$$

where $\gamma$ is the cluster surface-tension constant and $\mathbf{r}_{\mathrm{cl}}$ is the radius of the cluster. The point at which an ion is just in contact with the cluster surface corresponds to zero force. Further, this force is active for all anion motions, but only acts on cations when the cation position is at or beyond the initial cluster boundary (i.e., outer cation of Fig. 1). The corresponding variation in energy is given by

$$
\Delta U_{s}^{2}=\gamma \pi\left[\|\mathbf{p}\|-r_{\mathrm{cl}}\right]^{2} \text {. }
$$

\section{B. Implementation}

The model is initiated by uniformly distributing the anions on the surface of a spherical cluster, where the number of ionic groups, their size, and the cluster size are all input parameters. The cations, where their number, their charge, and their size are all input parameters, are then randomly distributed within the cluster.

Once distributed, the anions and the cations are allowed to interact with one another until equilibrium positions are established. Only after the equilibrium positions are estab- 
TABLE I. Input parameters, symbols, and values.

\begin{tabular}{lcl}
\hline \hline Parameter & Symbol & \multicolumn{1}{c}{ Value } \\
\hline Cluster radius & $r_{c l}$ & $2.105 \mathrm{~nm}$ \\
Maximum separation of ion pairs & $r_{\max }$ & $0.361 \mathrm{~nm}$ \\
Number of anions & $n_{\text {an }}$ & 26 \\
Anion radius & $r_{\text {an }}$ & $0.24 \mathrm{~nm}$ \\
Ion charge & $c$ & $\pm 1.602 \times 10^{-19} \mathrm{C}$ \\
Number of cations & $n_{\text {cat }}$ & $1-50$ \\
Cation radius (Na+) & $r_{\text {cat }}$ & $0.097 \mathrm{~nm}$ \\
Pendant chain stiffness & $k_{\text {pc }}$ & $0.0125 \mathrm{~N} / \mathrm{m}$ \\
Effective dielectric constant & $\epsilon$ & 78 \\
Surface energy constant & $\gamma$ & $0.0729 \mathrm{and} 0.0036 \mathrm{~N} / \mathrm{m}$ \\
Perturbation range & $\alpha_{\text {per }}$ & $0^{\circ}$ \\
Maximum step size & & $0.002 \mathrm{~nm}$ \\
Externally applied electric field & $\mathbf{E}_{\mathbf{a}}$ & $1 \times 10^{4} \hat{k} \mathrm{~V} / \mathrm{m}$ \\
\hline
\end{tabular}

lished ("equilibrium 1") is an external electric field applied. In response to this field, the ions are again allowed to find equilibrium ("equilibrium 2"). Table I provides the base line input parameters which correspond to Nafion ${ }^{\circledR}$ (DuPont) fully solvated with water and with sodium as the cation. All but two of the values provided in Table I are based on Datye and co-workers. ${ }^{13,14}$ The exceptions are the values for the dielectric constant and surface energy constant, where these are based on typical values for water; ${ }^{24-26}$ application of these constant, macroscopically determined values represent a simplifying assumption. ${ }^{20}$

The foregoing development of force and energy expressions relies largely on continuum (macroscale) concepts, and subsequently, the macroscopically determined surfacetension coefficient, $\gamma$, is applied. However, theoretical and computational studies on the length scale of nanometers presents an interesting challenge in that it is not truly continuum in nature, nor is it quantum. Moreover, this is the length scale studied in molecular dynamics, where quasicontinuum balland-spring approaches are typically applied, ${ }^{27,28}$ as has been done in this effort. Recognizing that the constant surfacetension coefficient derives from the intermolecular forces of a pure substance, one should expect that this constant will be locally altered when a single cation or anion group is acting upon the cluster surface. A detailed study of this effect is beyond the scope of this work; however, an indirect assessment $^{20}$ illustrates that application of the macroscopically determined value for cluster surface-tension constant, $\gamma_{b}$ leads to "stiff" surface response while application of $0.05 \gamma_{b}$ is the largest value for which the pendant chain force may have any significant impact on cluster size and response, and thus allows further insight into the role of each of the key parameters (cluster surface, pendant chain, electrostatic, and external field effects).

Because initial ion distributions are random, a large number of cases must be considered in order to adequately assess trends. In the parametric studies considered here, 1000 runs of each case are considered. ${ }^{20}$

\section{PARAMETRIC STUDIES}

Three parametric studies are considered: variation in pendant chain stiffness, introduction of various energy barri-

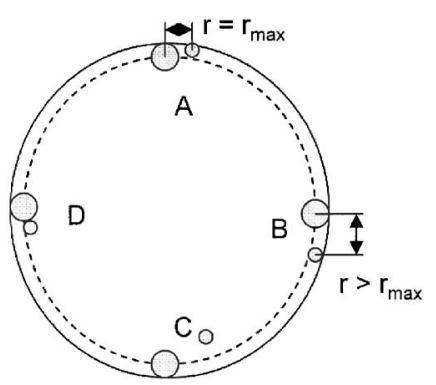

FIG. 2. (a) An ion pair, with an outer cation. (b) An unpaired anion and cation, with an outer cation. (c) An unpaired anion and cation. (d) An ion pair.

ers, and introduction of charge imbalance. Each of these cases is considered for two cluster surface-tension constants: $\gamma_{b}$, as noted in Table I, and $0.05 \gamma_{b}$.

For clarity and brevity, predictions for each study are provided in graphical form showing only the equilibrium 1 response (the first equilibrium reached based on the randomly initialized state). Any variations in response due to the application of external field are described in the text. While additional figures are presented where warranted, in all parametric studies there are graphs displaying the number of cations positioned at the outer extremes of the cluster (outer cations), the number of anion-cation pairs ("pairs"), and the average cluster radius $\left(r_{\text {ave }}\right)$. The significance of each of these categories is as follows.

It is assumed that cations positioned at the outer extremes of the cluster facilitate transport, either by leaving the cluster when given the appropriate stimulus, or by occupying an anion in a pair, thus opening a neighboring region for another cation to pass. For a cation to be identified as having an outer position its radial position value must at least equal $r_{\mathrm{cl}}-r_{\text {an }}$ (Fig. 2).

Anion-cation pairs are considered to address the common assumption of complete pairing. In all studies considered here, 26 anions are used. Thus, if 26 cations are also present, then traditional approaches assume that 26 pairs will form. Unpaired cations are assumed to be more likely to transport to a neighboring cluster than paired cations.

Cluster size and shape are taken to be dictated by anion position. If all anions have the same radial magnitude and are uniformly distributed, the cluster will be perfectly spherical. Any anion motion away from this perfect case will introduce variations to the classically assumed spherical shape. Thus, the cluster size and shape are considered in terms of the average radial position of the anions. It is found that the standard deviation away from this average radius is consistently two or more orders of magnitude smaller than the prediction itself, and is thus omitted from reporting.

\section{A. Case 1: Variation of pendant chain stiffness, $\gamma_{b}$}

This study (Fig. 3) considers the effect of varying the pendant chain stiffness for the case of surface-tension constant of $\gamma_{b}$ (Table I). It is first noted that pendant chain stiffness has moderate impact on the predicted number of ion pairs and outer cations. The number of predicted pairs increases for the increased pendant chain stiffness. Decreasing 
Outer Cations

$\gamma_{\mathrm{b}}, \Delta 0.05 \cdot \gamma_{\mathrm{b}}$



(a)

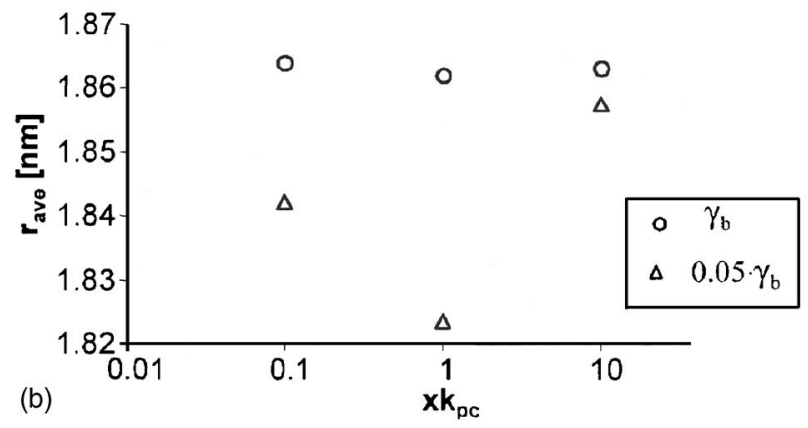

FIG. 3. Variation of pendant chain stiffness. (a) The predicted number of ion pairs $\quad(1.4<$ standard deviation $<2.6)$ and outer cations $(1.2$ $<$ standard deviation $<2.2$ ). (b) The predicted average cluster radius.

pendant chain stiffness predicts the largest number of outer cations of the parametric studies conducted in this work; Fig. 4 illustrates a single view of the initialized and final states for a sample case. This figure not only highlights the increasing number of outer cations at equilibrium, but also predicts the significant anion motion in the formation of "ion chains." Despite significant motion of all ions, pendant chain stiffness has virtually no effect on the predicted cluster size.

The only detected effect to the application of an external electric field is for the case of the softest $k_{\mathrm{pc}}$, where the average number of outer cations drops from 8.7 to 7.8 . Because the prediction is counterintuitive, the cations with $\pm z$ positions are considered separately; the majority $(\sim 65 \%)$ of the ions moving toward the interior are those with $-z$ coordinates, and are thus moving in the direction of the applied field. Also of interest, the typical internal fields are on the order of $10^{8} \mathrm{~V} / \mathrm{m}$, but for the softest case these fields drop by almost an order of magnitude.

\section{B. Case 2: 26 Variation of pendant chain stiffness, $0.05 \gamma_{b}$}

To allow closer inspection of the effect of $k_{\mathrm{pc}}$, this study repeats that of case 1, but for the case of the reduced cluster surface energy. Referring again to Fig. 3, the pendant chain stiffness now affects the predicted cluster size. Moreover, there is an unexpected prediction of increased cluster size for decreased pendant chain stiffness. In comparison to the $\gamma_{b}$ case (case 1), variation of $k_{\mathrm{pc}}$ again has moderate impact on the predicted number of ion pairs, but a lesser impact is noted on the predicted number of outer cations. Only the
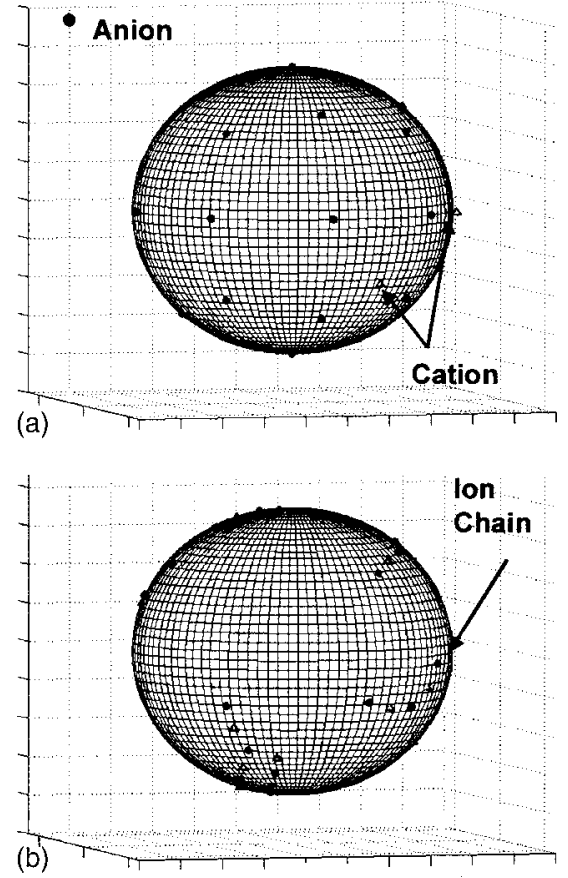

FIG. 4. Sample prediction of ions at the outer positions for parameters $\gamma_{b}$ and $0.1 k_{\mathrm{pc}}$. (a) Initialized state with two outer cations shown. (b) Predicted equilibrium state showing appearance of additional outer cations and the formation of an "ion chain."

highest stiffness appears to impact the predicted number of outer cations, and in a reversed sense from the $\gamma_{b}$ case.

Again, the only notable effect to the application of an external electric field is a reduction in the predicted number of outer cations for the softest stiffness; in this case when the $\pm z$-positioned cations are considered separately, it is found that the slight majority $(\sim 55 \%)$ of the ions moving toward the interior are moving in the direction of the applied field. The typical internal fields continue to be on the order of $10^{8} \mathrm{~V} / \mathrm{m}$ for the two stiffer pendant chain cases, but drop by almost an order of magnitude for the softest case (again).

\section{Case 3: Introduction of an energy barrier, $\gamma_{b}$}

By making energy reduction the only criterion for accepting a proposed ion step, losses such as the dissipation associated with pendant chain reconfiguration are neglected. In order to qualitatively assess the impact of this assumption on model predictions, introduction of various barrier energies is considered. The barrier energies tested, as noted in Fig. 5, are arbitrarily selected as $5 \times 10^{-27}, 5 \times 10^{-24}$, and 1 $\times 10^{-23} \mathrm{~J}$, where the energy drop associated with ion steps in this model can be as high as $10^{-22} \mathrm{~J}$.

The lowest-energy barrier considered, $5 \times 10^{-27} \mathrm{~J}$, is the only case where any response is predicted to the application of an external electric field (equilibrium 2); however, the results predicted are negligibly different from the case with no barrier. For the two higher barrier energies, the model has no response to the application of an external electric field (all ion steps are rejected in the first iteration). However, for these higher-energy barrier cases there is an increase in the predicted number of outer cations as compared to the zero barrier case. The increase is pronounced at the intermediate 
Outer Cations

$\approx \gamma_{b}, \Delta 0.05 \cdot \gamma_{b}$

Pairs

$* \gamma_{b}, \circ 0.05 \cdot \gamma_{b}$
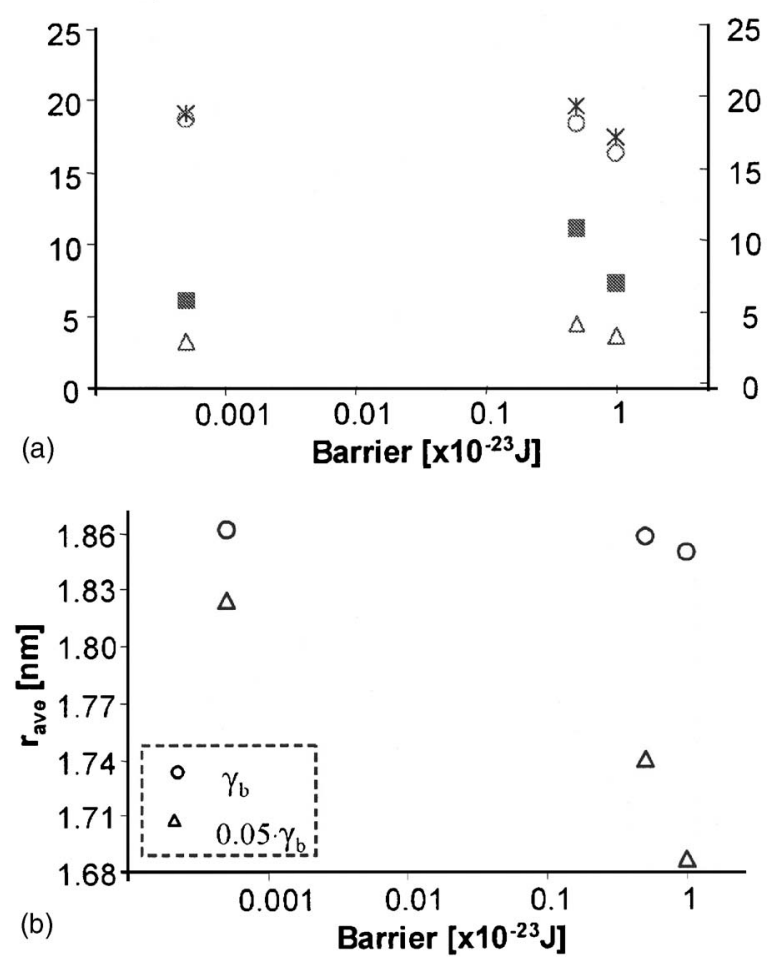

FIG. 5. Introduction of barrier energy. (a) The predicted number of ion pairs $(1.8<$ standard deviation $<2.2)$ and outer cations $(1.4<$ standard deviation $<1.6$ ). (b) The predicted average cluster radius.

barrier of $5 \times 10^{-24} \mathrm{~J}$. Conversely, the predicted number of ion pairs peaks and then drops below the predictions with no barrier. Lastly, only small variations in the predicted cluster size are observed.

\section{Case 4: Introduction of an energy barrier, $0.05 \gamma_{b}$}

When the impact of various energy barriers is considered for the $0.05 \gamma_{b}$ case (Fig. 5), the same trends noted for case 3 in the predicted number of outer cations are again observed. Further, the same trends in response to the application of external electric field are again observed as compared to case 3 . However, the predicted number of ion pairs and average cluster radius both decrease as the barrier energy increases.

\section{E. Case 5: Charge imbalance, $\gamma_{b}$}

It is well established that ion transport occurs in ionic polymers under the application of external electric field. This study (Fig. 6) considers the cluster response to the resulting charge imbalance. The first significant prediction is that there is no case, not even those of extreme imbalance, where ion pairing is complete. Next, the number of predicted outer cations increases faster than the charge imbalance itself. Lastly, the introduction of charge imbalance has a modest impact on the predicted cluster size, where the charge-balanced case (26 cations) is the smallest. Figure 7 graphically illustrates the predicted charge density, where the volume considered is based on the position of the ion with the largest radial component. When comparing Figs. 7(a) and 7(b), a distinct linear
Outer Cations

Pairs

$\gamma_{b}, \triangle 0.05 \cdot \gamma_{b}$

* $\gamma_{b}, 0.05 \cdot \gamma_{b}$

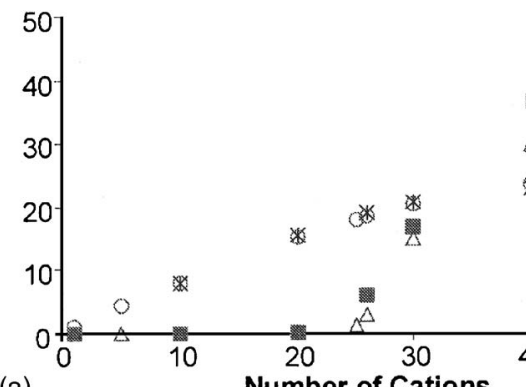

(a)

Number of Cations

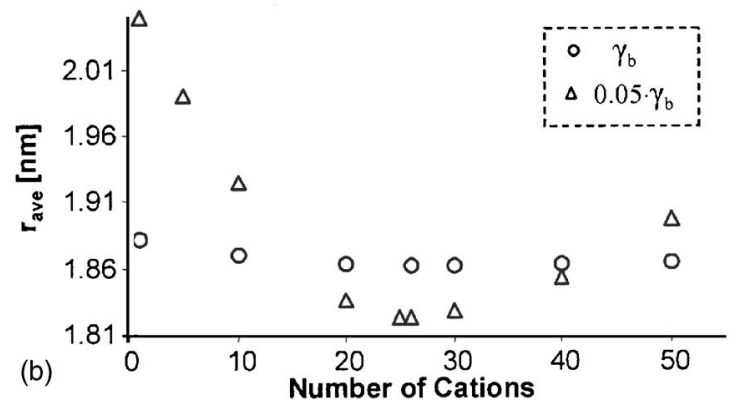

FIG. 6. Introduction of charge imbalance. (a) The predicted number of ion pairs $\quad(1.2<$ standard deviation $<1.9)$ and outer cations $(0.4$ $<$ standard deviation $<1.4$ ). (b) The predicted average cluster radius.

trend is observed in the charge density with respect to charge imbalance, while the volume over which it is calculated is highly nonlinear. Further, this linear trend is directly related to the application of a spherical volume dictated by the ion with the largest radial position rather than the average radial value.
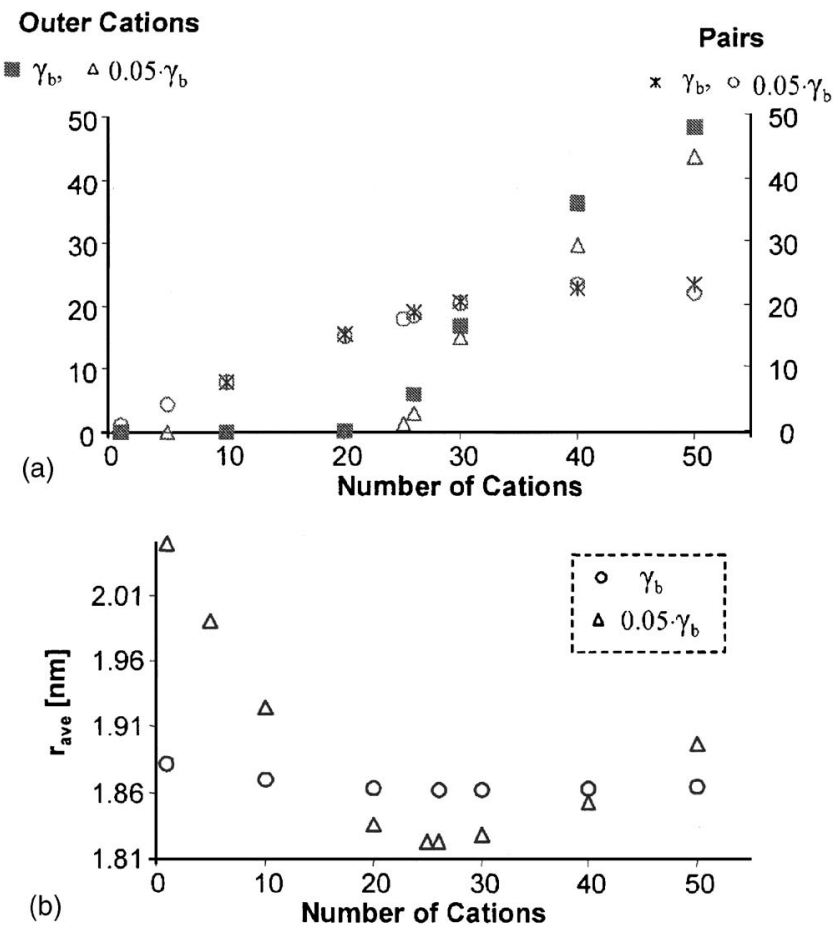

FIG. 7. (a) Variation in predicted charge density with charge imbalance for $\gamma_{b}$ cluster surface case; line provided to guide the eye. (b) Variation of predicted spherical volume-based $r_{\max }$ and $r_{\text {ave }}$, where $r_{\max }$ is used in the calculation of charge density and is the ion with greatest radial magnitude. 


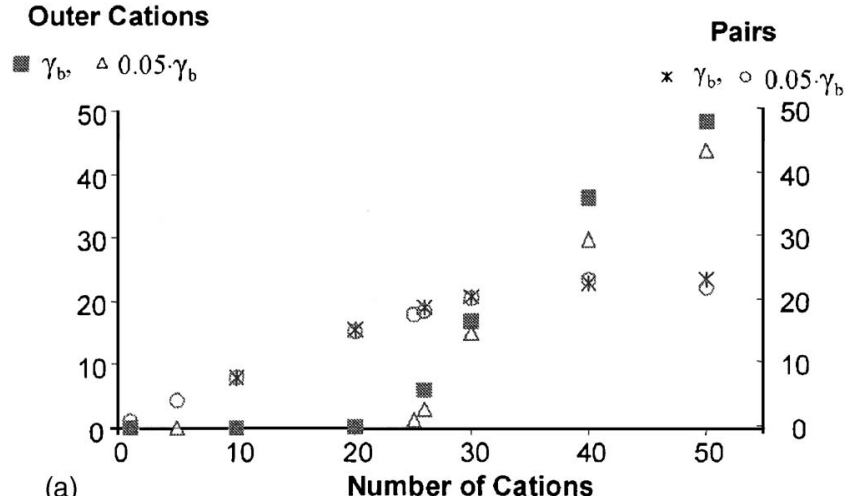

(a)

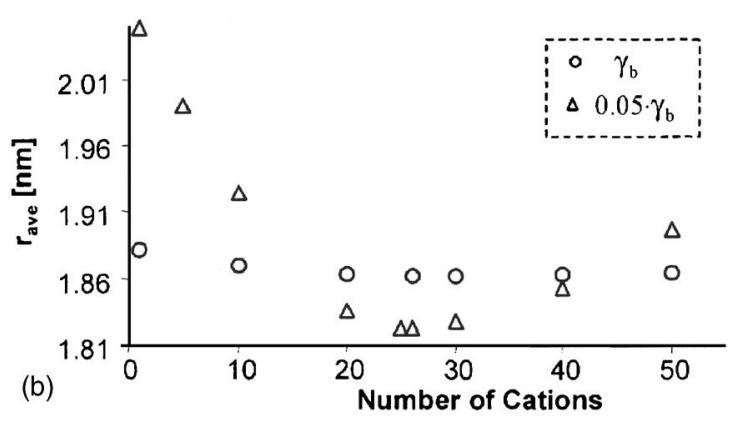

FIG. 8. (a) Variation in predicted charge density with charge imbalance for $0.05 \gamma_{b}$ cluster surface case; line provided to guide the eye. (b) Variation of predicted spherical volume-based $r_{\max }$ and $r_{\mathrm{ave}}$, where $r_{\max }$ is used in the calculation of charge density and is the ion with greatest radial magnitude.

\section{F. Case 6: 26 charge imbalance, $0.05 \gamma_{b}$}

This study (Fig. 6) considers the cluster response to charge imbalance for $0.05 \gamma_{b}$. In all but the cation-depleted case, ion pairing is predicted to be incomplete at equilibrium. All of the same trends discussed for case 5 are repeated, but have magnitudes which are either translated or magnified. For instance, the same trend in the predicted number of outer cations is observed for this case, but with fewer total at each increment. Similarly, the charge-density predictions (Fig. 8) and cluster size predictions display identical trends, but with variation in magnitude. For instance, the variation in the average cluster size $\left(r_{\text {ave }}\right)$ for the case of a single cation displays significant increase from the charge neutral case (26 cations), as illustrated in Fig. 9.

\section{DISCUSSION}

This discussion will skip the bulk of the "intuitive" predictions, such as reduced anion movement in response to increased pendant chain stiffness. The less intuitive, or sometimes counterintuitive predictions are discussed and include general tendencies, as well as case by case observations. Further, in some instances the discussion will relate the current predictions to those of Weiland and Leo. ${ }^{20}$

\section{A. General tendencies}

The first significant observation from these model predictions is the pervading tendency for incomplete ion pairing, while simultaneously a nontrivial number of cations are predicted to assume equilibrium positions at the outer extremities of the cluster. When taken together, this suggests
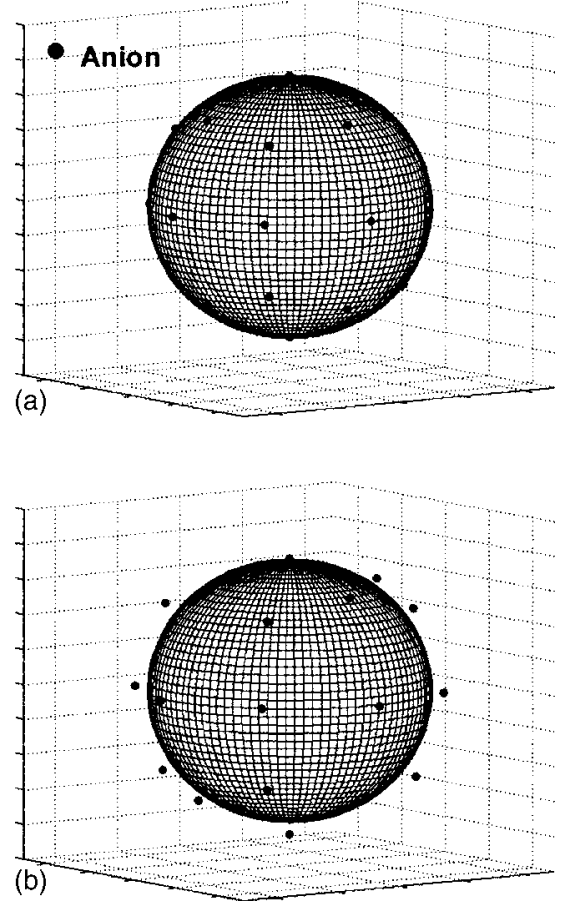

FIG. 9. Sample prediction of variation in cluster size based on anion positions for parameters $0.05 \gamma_{b}$ and one internal cation. (a) Initialized state. (b) Predicted equilibrium state showing anion position relative to initialized position (represented by sphere).

that cluster equilibrium states are predisposed to cation transport. This is consistent with the transport mechanism proposed by Datye et al. ${ }^{13}$ where the nontrivial number of pairs at the cluster surface still provide the impetus for transport, but with the added understanding of how it is possible that free cations are available for transport. The net effect of this prediction is to suggest that the larger length scale models which assume complete pairing will tend to underpredict transport. This result is consistent with and reinforces the results of the previous studies. ${ }^{20}$

Further, considering both the previous studies ${ }^{20}$ and the current studies, the number of predicted pairs for the chargebalanced cases is relatively insensitive to variations in cluster parameters. For all cases except the energy barrier studies the predicted number of pairs is between 18.6 and 20.6; for the energy barrier cases, where the barriers are chosen arbitrarily to assess trends and do not necessarily correspond to physical predictions, this range shifts to 16.2-19.1. Moreover, even for the charge-imbalanced cases with excess cations, the maximum predicted number of pairs only increases to 23.6 of the possible 26 pairs. It is believed that this prediction is related to the inclusion of the Pauli repulsion effect addressed in the electrostatic interaction [Eq. (6)] in combination with restricted anion mobility.

The number of cations predicted at the outer extremes of the cluster is sensitive to variations in cluster parameters, with a low prediction of 3.1 and a high of 11.0. It is presumed in this effort that a large number of cations positioned at the outer positions corresponds to a state that is transport facilitated. The rationale is that an outer cation is positioned favorably for transport to a neighboring cluster; in the event that cation is inhibited from transport by pairing it opens a 
neighboring vicinity for other cations to transport with minimal interference. The parameters having the most dramatic impact on the predicted number of outer cations are cluster surface-tension constant, $\gamma_{,}{ }^{20}$ pendant chain stiffness, $k_{\mathrm{pc}}$, and barrier energy. In general, a stiffer $\gamma$ leads to increased cations at the surface, while the impact of $k_{\mathrm{pc}}$ depends also on $\gamma$. The value in this prediction is that by varying both (i) the solvent or the cation introduced (and thereby altering $\gamma$ ), and (ii) the ionomer fabrication (altering $k_{\mathrm{pc}}$ ), significant control over this parameter is envisioned. The challenge here is that introduction of alternate solvents or cations, will necessarily impact the self-organized cluster size and shape. This value is one over which little control may be exercised at present. To establish reliable control over the number of cations positioned at the outer extremities of the cluster, and facilitate transport, an optimization of various fluid/polymer interactions would be required.

One of the goals of this effort is to investigate the impact of the classically assumed spherical cluster shape. In the studies considered, even when significant ion movement results in the average cluster radius changing by almost $10 \%$ (the cation-depleted case of Fig. 9 or nonuniform anion distribution $\left.^{20}\right)$, the standard deviation in position from anion to anion is two orders of magnitude lower than the prediction itself. Thus, for an initialized spherical shape, even under significant ion motion, the shape remains essentially spherical. It is of course understood that the assumption of spherical clusters, even as an initialized condition, is an idealized assumption as compared to the anisotropic nature of actual clustering; however, in the absence of a universally accepted morphological model, ${ }^{29}$ it is assumed that this basis remains the most practical of the available options.

Some of the cases reported reduced internally generated electric fields. Because the result is unexpected, it is reported. However, because these calculations are done only at six specific points in space (at $x, y$, and $z= \pm R_{\mathrm{cl}}$ ), it is possible that the prediction is not general enough to be treated as a global prediction. It is, of course, conceivable that significantly different electric fields will be generated at points exterior to the cluster based on the cluster state.

\section{B. Transport (electric field and charge imbalance response)}

In light of transport, the inability of this model to predict significant variations in cluster state upon the application of external field has been explored in order to be thorough, but the result is not entirely unexpected. Because the current model offers no cation transport paths, while their existence is implicitly assumed in allowing the clusters to expand/ contract, none of the cations available for transport are able to move a significant distance. Thus, the second iteration associated with the application of field likely serves only to refine the predicted low-energy step via the application of the reduced step size. What is significant, however, is the absence of a predicted cluster shape change; it can be shown that this continues to be true even in the presence of increased externally applied electric fields consistent with the dendritic nature of electrode morphology. ${ }^{19,30}$ This is contrary to an earlier hypothesis of the reformation of clusters to a penny shape. ${ }^{17}$

While the construction of this model does not directly allow for ion transport, it is addressed indirectly by considering the cluster state for the case of charge imbalance. Two issues from the charge imbalance study warrant discussionthe variation in cluster radius and the corresponding variation in charge density.

First, with respect to variation in cluster radius, it is noted that the charge-balanced case takes on the smallest cluster size, while the cation-depleted case takes on the largest cluster size; this is consistent with the works of Nemat-Nasser. ${ }^{8,31}$ This result not only supports the supposition of the formation of boundary layers at the electrodes, it also supports the supposition that differential expansion due to electrostatic effects may be playing a significant role in the actuation of specific IPMCs.

In addition, it is because the cluster size is nonlinearly variable that the predicted linear charge density with respect to charge imbalance is interesting. As noted earlier, the volume used for charge-density calculation is spherical and based on the position of the ion with largest radial component, rather than $r_{\text {ave }}$. Figures 7 and 8 illustrate the variation in calculated volume for these two cases. (Naturally, calculated charge-density values would be much lower if considered over the surrounding charge neutral region as well.)

When Figs. 7(a) and 8(a) are compared, the linear response is a function of both charge imbalance and surfacetension stiffness. While not tested, it stands to reason that this relationship will be a function of multiple model parameters such as ion size and pendant chain stiffness. No physical explanation is currently offered for the cation reorganization necessary to predict this linear trend.

\section{Pendant chain stiffness}

In case 1 the base line cluster surface tension, $\gamma_{b}$, is applied while pendant chain stiffness is varied. In this case variation of pendant chain stiffness has negligible predicted effect on the cluster shape and size. This is because the relative stiffness of the cluster surface overpowers the pendant chain effect. However, in case 2, where the surface tension of $0.05 \gamma_{b}$ is applied, the prediction for the softest pendant chain case is counterintuitive. In all other cases, the tendency of the cluster is to contract from its initialized state; thus one would expect more contraction when pendant chain stiffness is reduced. However, the reverse is predicted; as compared to the base line pendant chain stiffness, less contraction is observed for the very soft case. It is proposed that the result is due to the interplay of pendant chain stiffness and electrostatic effects as illustrated by "ionic chain" formation shown in Fig. 4. It has been proposed that the effect of pendant chain resistance is to rotate the ion pairs to positions that approach normality with the surface while in the absence of these effects the pairs will tend to be tangential to the cluster surface. ${ }^{13}$ This prediction further suggests that the reduced stiffness also facilitates pair alignment on the surface. This prediction results from the initialized random placement of 
cations, where this initialized state is a reasonable estimate for the way in which cations are introduced to a cluster during the fabrication process. This prediction also illustrates that even when anion position is "encouraged" to maintain an outer spherical shell at the cluster surface via a large cluster surface tension, electrostatic effects alone do not lead to the prediction of a nested cation sphere; however, it still stands to reason that electrostatic effects lead to a significant contribution to the internal cluster pressure.

Next, the variation in trends observed for the predicted number of outer cations and ion pairs is considered. For the $\gamma_{b}$ case (case 1), the predicted number of outer cations decreases while the predicted number of pairs increases as the pendant chain stiffness increases. This case suggests that softer pendant chain values facilitate transport. However, for the $0.05 \gamma_{b}$ case (case 2), the predicted number of outer cations and ion pairs are at local minima and maxima, respectively, in comparison to variations in pendant chain stiffness. Thus for case 2 (and unlike case 1), the prediction suggests that there is an ideal, or optimized pendant chain stiffness. This suggests that the cluster surface-tension effects dominate over the pendant chain stiffness effects. This conclusion is drawn because reducing the cluster surface tension always predicts the same trend, namely, a decrease in the number of outer cations and ion pairs. ${ }^{20}$ This is a rather interesting prediction because cluster surface tension impacts only radial motion, while pendant chain response is to all anion movement. However, it is consistent with the postulate that the nature of the cation plays a dominant role in IPMC actuation, ${ }^{31}$ given that variation in the cation is expected to have varying influence on the effective cluster surface constant.

\section{Energy barriers}

This model assumes reversible behavior. However, it is expected that dissipation will occur in response to ion motion; for instance, in response to the reconfiguration of the pendant chain and viscous losses. Therefore, in order to address this reality, a few arbitrary energy barriers are introduced corresponding, say, to increasingly viscous solvents. Increases in the barrier energy lead to predictions of further decreased cluster size. Predicted cluster size is dramatically decreased for the largest barrier and $0.05 \gamma_{b}$; it is proposed that this barrier is unnaturally large for this case. However, the $5 \times 10^{-24}$ - J barrier appears to further facilitate cation migration based on the increased number of cations predicted at the outer extremities of the cluster. Thus, it appears that application of the assumption of reversible ion motion will tend to underpredict the transport-facilitated state of a cluster. Clearly this is a point worthy of further study, but is best implemented in conjunction with electrodynamic effects.

It should be noted that this set of results on the impact of barrier energy must be considered with caution, and should only be used for qualitative assessment because the model is not modified to account for the effects of varying step lengths (for instance, when the step length is reduced because of ion overlap conditions) as well as the issue of assigning different barriers for different mechanisms.

\section{CONCLUSIONS}

In this work the impact of assuming spherical cluster shapes with uniform ion distribution is investigated in the presence of pendant chain stiffness variations, energy barriers, and charge imbalance. The model predicts that, due to inhibited anion motion via elastic and surface energy effects, combined with Pauli repulsion, ion paring is rarely complete, thus facilitating cation transport. Overall, this model suggests that application of the assumption of uniform charge distribution over a spherical cluster shape will tend to underpredict the observed macroscopic transport response. The initialized spherical cluster shape is essentially maintained throughout the various case studies, including during the application of locally high external electric field. Further, the methodology employed in this study provides a potential mechanism for informed control over transport properties by manipulating pendant chain stiffness in conjunction with cluster surface tension, where pendant chain stiffness plays an important but secondary role to cluster surface tension.

Transport effects are studied indirectly through the introduction of cluster charge imbalance. Net cluster size tends to increase for clusters that are cation depleted; cluster size for a surplus of cations also tends to increase, but to a lesser extent. Thus, the electromechanical bending response for the base line case studied here (hydrated Nafion in salt form with $\mathrm{Na}^{+}$cation) may have significant electrostatic contribution. This is consistent with the formation of anode and cathode boundary layers; no assertions are made with regard to the role of electrostatics versus solvent transport for the general case. Further, despite a nonlinear variation in the cluster size with respect to charge imbalance, the charge-density predictions are linear with respect to charge imbalance.

\section{ACKNOWLEDGMENT}

The authors would like to acknowledge the support of the National Science Foundation Grant No. CMS-0093889.

${ }^{1}$ K. Sadeghipour, R. Salomon, and S. Neogi, Smart Mater. Struct. 1, 172 (1992).

${ }^{2}$ K. Oguro, Y. Kawami, and H. Takenaka, Osaka Kogyo Gijutsu Shikensho Kiho 43, 21 (1992).

${ }^{3}$ Y. Osada and M. Hasebe, Chem. Lett. 1985, 1285.

${ }^{4}$ M. Irie, Macromolecules 19, 2890 (1986).

${ }^{5}$ T. Shiga and T. Kurauchi, J. Appl. Polym. Sci. 39, 2305 (1990).

${ }^{6}$ M. Shahinpoor, Smart Mater. Struct. 1, 91 (1992).

${ }^{7}$ M. Shahinpoor, Y. Bar-Cohen, J. O. Simpson, and J. Smith, Smart Mater. Struct. 7, R15 (1998).

${ }^{8}$ Electroactive Polymer (EAP) Actuators as Artificial Muscles-Reality, Potential, and Challenges, edited by Y. Bar-Cohen (SPIE-The International Society for Optical Engineering, Bellingham, Washington, 2001).

${ }^{9}$ M. Mojarrad and M. Shahinpoor, Proc. SPIE 2779, 1012 (1996).

${ }^{10}$ S. Nemat-Nasser, J. Appl. Phys. 92, 2899 (2002).

${ }^{11}$ K. Farinholt, K. Newbury, M. Bennet, and D. Leo, 2002 An Investigation into the Relationship Between Charge and Strain in Ionic Polymer Sensors, First World Congress on Biomimetics and Artificial Muscles, Albuquerque, NM, 9-11 December 2002 (unpublished).

${ }^{12}$ W. Y. Hsu and T. D. Gierke, Macromolecules 15, 101 (1982).

${ }^{13}$ V. K. Datye, P. L. Taylor, and A. J. Hopfinger, Macromolecules 17, 1704 (1984).

${ }^{14}$ V. K. Datye and P. L. Taylor, Macromolecules 18, 1479 (1985).

${ }^{15}$ A. Lehmani, S. Durand-Vidal, and P. Turq, J. Appl. Polym. Sci. 68, 503 (1998).

${ }^{16}$ P. G. de Gennes, K. Okumura, M. Shahinpoor, and K. J. Kim, Europhys. Lett. 50, 513 (2000) 
${ }^{17}$ J. Y. Li and S. Nemat-Nasser, Mech. Mater. 32, 303 (2000).

${ }^{18}$ S. Nemat-Nasser and J. Y. Li, J. Appl. Phys. 87, 3321 (2002).

${ }^{19}$ L. M. Weiland and D. J. Leo, Smart Mater. Struct. 13, 323 (2004).

${ }^{20}$ L. M. Weiland and D. J. Leo, J. Appl. Phys. 97, 013541 (2005).

${ }^{21}$ J. A. Stratton, Electromagnetic Theory (McGraw-Hill, New York, 1941).

${ }^{22}$ A. L. Ruoff, Introduction to Materials Science (Robert E. Krieger, Huntington, NY, 1979).

${ }^{23}$ L. H. van Vlack, Elements of Materials Science and Engineering, 5th ed. (Addison-Wesley, Reading, MA, 1985).

${ }^{24}$ Dr. R. B. Dooley, Release on Surface Tension of Ordinary Water Substance, The International Association for the Properties of Water and Steam (Electric Power Research Institute, Palo Alto, CA, 1994).

${ }^{25}$ Dr. R. B. Dooley, Release on the Static Dielectric Constant of Ordinary Water Substance for Temperatures from $238 \mathrm{~K}$ to $873 \mathrm{~K}$ and Pressures up to $1000 \mathrm{MPa}$, The International Association for the Properties of Water and Steam (Electric Power Research Institute, Palo Alto, CA, 1997)

${ }^{26}$ J. N. Murrell and A. D. Jenkins, Properties of Liquids and Solutions, 2nd ed. (Wiley, New York, 1994).

${ }^{27}$ M. Fermeglia, S. Pricl, and G. Longo, Chem. Biochem. Eng. Q. 17, 19 (2003).

${ }^{28}$ K. Kremer and F. Mller-Plathe, MRS Bull. 26, 205 (2001).

${ }^{29}$ K. A. Mauritz and R. B. Moore, Chem. Rev. (Washington, D.C.) 104, 4535 (2004).

${ }^{30}$ B. J. Akle, K. B. Wiles, D. J. Leo, and J. E. McGrath, Effects of Electrode Morphology on the Performance of BPSH and PATS Ionic Polymer Transducer, Proceedings of the SPIE 11th Annual Symposium on Smart Structures and Materials, San Diego, California, 2004 (SPIE-The International Society for Optical Engineering, Bellingham, Washington, 2004), Vol. 5385 .

${ }^{31}$ S. Nemat-Nasser, J. Appl. Phys. 92, 2899 (2002). 